EESTI NSV TEADUSTE AKADEEMIA TOIMETISED, 22, KOIDE

KEEMIA* GEOLOOGIA. 1973, NR. I

ИЗВЕСТИЯ АКАДЕМИИ НАУК ЭСТОНСКОП ССР. ТОМ 22 ХИМИЯ * ГЕОЛОГИЯ. 1973, № 1

удК $51: 550.824-552.123$

Ю. ПААП

\title{
РАЗМЕРНЫЕ ГРАНУЛОМЕТРИЧЕСКИЕ ШКАЛЫ И ИХ ТЕОРИЯ
}

Множество известных к настоящему времени гранулометрических шкал широко используется во многих геологических исследованиях. Поскольку в каждом конкретном случае приходится выбирать одну из возможных шкал, это порождает известные трудности во взаимопонимании исследователей (неоднозначное использование терминов), но особенно при сопоставлении и обобщении количественных данных, полученных разными исследователями.

Первую известную и поныне используемую гранулометрическую шкалу предложил Дж. Адден (Udden, 1898). К настоящему времени стали обшеизвестными гранулометрические шкалы Аттерберга (Atterberg, 1905), Уентворта (Wentworth, 1922), десятичная метрическая шкала (впервые предложена К. Гопкинсом в 1899 году, по Krumbein, Pettijohn, 1938) и разные логарифмические шкалы (Krumbein, 1934; Батурин, 1943). Наряду с этими существуют десятки других шкал, менее известных или применяемых только определенным кругом специалистов для конкретных целей (исследование почв, строительных или формовочных материалов, керамического и других видов промышленного сырья и т. п.). Назвать какую-нибудь шкалу «общепринятой», как это, например, делает В. Шванов (1969), невозможно. В Советском Союзе большинство исследователей пользуется десятичной метрической шкалой (Ершова, 1962; Раукас, 1964; Дмитриева и др., 1968 и др.) или разными ее вариантами с непостоянным отношением конечных размеров классов (Рухин, 1969; Шванов, 1969 и многие др.). В Западной Европе (кроме Англии) и в Скандинавии большинство авторов применяют шкалу Аттерберга (Köster, 1960, 1964; Müller, 1964 и др.), а в США почти все исследователи предпочитают шкалу Уентвопта (Krumbein, Pettijohn, 1938; Pettijohn, 1949; Krumbein, Graybill, 1965; Griffiths, 1967; Tanner, 1969 и др.), которая применяется и в некоторых социалистических странах (Konta, 1968 и др.).

\section{Назначение гранулометрических шкал}

Общепринятое содержание понятия «гранулометрическая шкала» (Ершова, 1962; Данбар, Роджерс, 1962; Рухин, 1969; Шванов, 1969; Griffiths, 1967; Tanner, 1969 и др.) неоднозначное. Это легко видеть по следующему примеру.

Гранулометрические шкалы Аддена, Уентворта и Аттерберга применяются для классификации осадочных частиц по раз м е р у и могут быть выражены в любых единицах длины (дюймах, мил- 
лиметрах, микрометрах и т. д.), при этом размер служит для точного и однозначного определения границ таксонов (Шарапов, 1966). При переходе от одной системы единиц к другой (если они взаимно трансформируемы) классификация и ее таксоны остаются прежними, изменяются только цифровые транскрипции границ классов. Поэтому наличие размерной шкалы является необходимым условием для создания града ционной (классификационной) гранулометрической шкалы; без установления однозначных цифровых границ подобная номенклатурная классификация лишена содержания. С другой стороны, гранулометрические шкалы на логарифмической основе (фи- и дзеташкалы Крамбейна и гамма-шкала Батурина) применяются для выр ажения размера частиц, особенно при математической или графической обработке данных анализа, и они являются самостоятельными. Поэтому их можно назвать размерными гранулометриче ски ми шкалами.

\section{Размерные гранулометрические шкалы}

Чаще всего для выражения размеров составляющих частей пород применяется линейный масштаб, однако точность таких измерений пропорциональна логарифму единиц размера. Практические пределы современного гранулометрического анализа охватывают промежуток от 0,0001 до 1000 мм (от коллоидных частиц до глыб пород), т. е. гранулометрический спектр охватывается порядком $10^{7}$. В природе встречаются отложения (например, морены), в которых одновременно присутствуют частицы с размерами всех порядков.

Возникшее в результате природных процессов, а также в результате раздробления распределение частиц по крупности является логари фм и ческим (Колмюгоров, 1941; Миддлтон, 1968). Здесь не доказывается, является ли это распределение логарифмически-нормальным, поскольку существеннее вывод, что физическому содержанию гранулометрического спектра соответствует не линейный, а логарифмический масштаб размерной гранулометрической шкалы.

\section{Логарифмические гранулометрические шкалы}

В настоящее время применяются следующие логарифмические гранулометрические шкалы: фи- и дзета-шкалы Крамбейна, гамма-шкала Батурина и пси-шкала автора (Паап, 1969). Выбор конкретной шкалы определяется исходя из принятой классификации, применяемых технических средств и методов анализа, существующих государственных стандартов и требований промышленности.

По сравнению с линейными приведенные логарифмические шкалы обладают следующими преимуществами:

1) соседние интервалы (деления) шкалы являются равными и возрастают в арифметической прогрессии, что намного упрощает и облегчает графическую обработку и представление результатов гранулометрии;

2) геометрическая основа (логарифмы при любом основании) шкалы позволяет выразить размеры частиц с одинаковой точностью в пределах всего гранулометрического спектра;

3) размерные границы классов выражаются относительно простыми числами, а при строгом соответствии градационной и размерной шкал целыми числами; 
4) выражение размера частиц в логарифмических единицах намного упрощает математическую обработку данных гранулометрии, включая применение ЭВМ;

5) логарифмические шкалы спответствуют физической природе гранулометрического состава пород, что облегчает создание физических моделей седиментации на статистической основе;

6) математическая основа логарифмических шкал позволяет полностью формализовать перевод размеров из одной шкалы в любую другую без промежуточных преобразований;

7) все основные деления приведенных шкал включены в государственные стандарты разных стран и используются при выпуске соответствующего оборудования.

Рассмотрим далее каждую из логарифмических шкал. Фи- и дзеташкалы были разработаны У. Крамбейном (Krumbein, 1934), который предложил для шкалы Уентворта следующее математическое преобразование:

$$
x=2^{-\varphi}, \varphi=-\log _{2} x,
$$

где $x$ - размер частиц в миллиметрах.

По аналогии с фи-шкалой он предложил преобразование и для шкалы Аттерберга, принимая за начальный размер 2 мм (максимальный диаметр капиллярного поднятия, принятый А. Аттербергом за начальную точку его шкалы)

$$
x=10^{\lg 2-6}=10^{0,301-\zeta}, \zeta=\lg 2-\lg x=0,301-\lg x .
$$

Так как в Советском Союзе шкалы Уентворта и Аттерберга не нашли распространения, В. Батурин (1943), следуя рассуждениям У. Крамбейна, разработал гамма-шкалу, которая согласуется с десятичной метрической шкалой,

$$
x=10^{-\frac{\gamma}{10}}, \gamma=-10 \lg x
$$

Все приведенные шкалы распространяются до бесконечности в обе стороны от центра шкалы. Однако использование этих шкал затрудняется, во-первых, тем, что они включают как отрицательные, так и положительные значения. Кроме того, выбранные центры шкал (за исключением дзета-шкалы) не совпадают с границами классов, применяемыми теми же авторами. Например, по шкале Уентворта размер песчаных частиц изменяется от $4 \varphi\left({ }^{1} / 64\right.$ мм $)$ до $-1 \varphi(2$ м. $)$. Определенные затруднения создает и то непривычное обстоятельство, что фи-, дзета- и гамма-шкалы являются обрашенными, т. е. значения шкал обратно пропорциональны величинам измеряемых объектов (например, 0,001 мм = $9,99 \varphi=3,301 \zeta=30 \gamma ;$ a $100 \mu M=-6,65 \varphi=-1,699 \xi=-20 \gamma)$.

Короче, недостатки приведенных шкал заключаются в 1) наличии отрицательных и положительных единиц; 2) обратной пропорциональности цифровых значений делений шкал измеряемым размерам объектов; 3) недостаточной обоснованности расположения центров шкал и в несовпадении их с границами используемых классов.

Несмотря на это приведенные шкалы находят широкое применение, особенно фи-шкала. Однако в Советском Союзе и во многих странах Европы ее распространению препятствует применение десятичной метрической шкалы, на использовании которой основаны не только методика и технические средства анализа, но и большинство государственных стандартов (ГОСТ 3584-53, DIN 1188 и 4188 и др.). 


\section{Теоретическая основа логарифмических гранулометрических шкал}

Изобразим на числовой оси (рис. 1) гранулометрический спектр, измеряемый положительным и единицами длины. При этом $x_{0}$ и $x_{n}$ являются конечными величинами. В соответствии с физической природой гранулометрического спектра числовая последовательность $x_{0}, x_{1}, x_{2}, \ldots$ 。

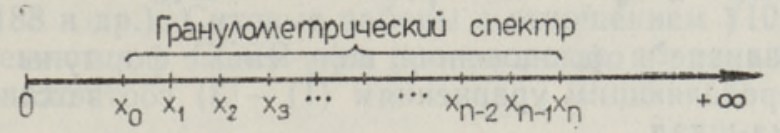

Рис. 1. Обобщенное изображение гранулометрического спектра на числовой оси.

Generalized representation of the grain-size spectrum on the number-axis.

$x_{n-1}, x_{n}$ образует геометрическую прогрессию, в которой, как известно, отношение каждого следующего члена к предыдущему остается постоянным

$$
\frac{x_{1}}{x_{0}}=\frac{x_{2}}{x_{1}}=\ldots=\frac{x_{n}}{x_{n-1}}=q
$$

( $q$ называется знаменателем прогрессии) и любой член которой можно вычислить по формуле

$$
x_{n}=x_{0} q^{n}(n=0,1,2, \ldots) .
$$

Если знаменатель геометрической прогрессии принять за единицу логарифмической шкалы и через $\Psi$ обозначить порядок шкалы, то получим

$$
\begin{gathered}
q^{\Psi}=\frac{x_{n}}{x_{0}}, \text { откуда } \\
\Psi=\log _{q} x_{n}-\log _{q} x_{0}, \quad x_{n}=q^{\Psi+\log _{q} x_{0}} .
\end{gathered}
$$

Значения $\Psi$ образуют арифметическую прогрессию с постоянной разностью $d=1$ и формулы (4) можно назвать о п ределя ющ и м и у а в нени я м логарифмической гранулометрической шкалы. Они включают все применяемые логарифмические шкалы как конкретные приложения метрической системы.

1. Фи-шкала образует геометрическую прогрессию со знаменателем $q=2$. Она является обратно пропорциональной к единицам измерения. В качестве начала У. Крамбейн принял $x_{0}=1$ мм. Следовательно, $\varphi=$ $=-\Psi_{\varnothing}$ и по формулам (4)

$$
\begin{gathered}
\Psi_{\odot}=\log _{q} x_{0}-\log _{q} x, \quad \text { откуда } \\
\Psi_{\oplus}=\log _{2} 1-\log _{2} x=-\log _{2} x, \quad x=q^{-\Psi+\log q x_{0}}=2^{-\Psi_{\varphi}+\log _{2} 1}=2^{-\Psi \Phi} .
\end{gathered}
$$

2. Дзета-шкала также образует убывающую геометрическую прогрессию со знаменателем $q=10$ и $x_{0}=2$ мм. Применяя формулы (4), получим

$$
\begin{gathered}
\Psi_{\tau}=\log _{q} x_{0}-\log _{q} x=\lg 2-\lg x=0,301-\lg x, \\
x=q^{-\Psi_{\zeta}+\log _{q} x_{0}}=10^{-\Psi_{\zeta}+\lg 2}=10^{0,301-\Psi_{\zeta}} .
\end{gathered}
$$


3. Гамма-шкала выражается убывающей геометрической прогрессией со знаменателем $q=\sqrt{10}$ и $x_{0}=1$ мм. По формулам (4) получим

$$
\begin{gathered}
\Psi_{\nu}=\log _{q} x_{0}-\log _{q} x=-\log _{\sqrt[10]{10}} x=-10 \lg x, \\
x=q^{-\Psi v+\log q x_{0}}=\sqrt[10]{10}{ }^{-\Psi_{\gamma+\log _{10}}{ }^{1}}=10^{-\frac{\psi_{\gamma}}{10}} .
\end{gathered}
$$

Исключая различие в обозначениях, полученные формулы (5)-(7) тождественны определяюшим уравнениям (1)-(3) соответственно для фи-, дзета- и гамма-шкал.

При помощи определяющих уравнений логарифмической гранулометрической шкалы можно вывести любую логарифмическую размер ную шкалу с заданным знаменателем прогрессии (основание шкалы), расположением центра (нуль шкалы) и направлением. Дробные единицы шкалы, которые обозначают соотношение границ фракций, определяются по уравнению

$$
\Delta \Psi=\sqrt[m]{q},
$$

где $m$ - требуемая дробность деления. Например, $1 / 4 \varphi=\sqrt[4]{2} ; 0,1 \zeta=\sqrt[10]{10}$; $1 / 2 \gamma=\sqrt{\sqrt{10}}=\sqrt[20]{10}$ и т. д. Как физический показатель $\Delta \Psi$ выражает детальность анализа.

\section{Пси-шкала}

Выше приводились основные недостатки применяемых логарифмических шкал. Основываясь на предлагаемой теории гранулометрических шкал, автор разработал новую шкалу, которая согласуется с десятичной метрической шкалой и включает все метрические опорные размеры других шкал, за исключением фи-шкалы. Принимая за основу определяющие уравнения (4), предложим следующие преобразования:

$$
\psi=\lg x-\lg x_{0}, \quad x=10^{\psi+\lg x_{0}} .
$$

Основная трудность заключается в определении начала шкалы. Выберем для этого размер $x_{0}=0,0001$ м. ис исхоя из следующих соображений:

1) этот размер является верхней границей настоящих коллоидных частиц;

2) при размерах меньше 0,0001 мм начинает сказываться влияние броуновского движения;

3) частицы диаметром меньше 0,0001 мм согласно закону Стокса практически не могут выпасть в осадок без участия процессов коагуляции;

4) размер 0,0001 мм на один порядок меньше практического нижнего предела гранулометрического анализа $(0,001-0,002$ мм).

Принимая $x_{0}=0,0001 \mathrm{mм}$, определяющие формулы для пси-шкалы приобретают вид

$$
x=10^{\psi+\lg 0,0001}=10^{\psi-4}, \quad \psi=\lg x-\lg 0,0001=\lg x+4 .
$$

В приведенном виде пси-шкала имеет все положительные качества других шкал, но лишена тех недостатков, которые были отмечены выше. 
При применении последовательной десятичной метрической градационной шкалы границам гранулометрических классов будут соответствовать целые значения делений пси-шкалы $(0,001 м \mu=1 \psi ; 0,01 м \mu=2 \psi ; 0,1 м \mu=$ $=3 \psi$ и т. д.). Например, детальности анализа $0,1 \psi$ соответствуют выпускаемые промышленностью сита (в СССР ГОСТ 3584-53, в ГДР и ФРГ DIN 1188 и 4188 и др.). Ситовые наборы с отношением $\sqrt[10]{10}=1,25(\Delta \Psi=$ $=0,1 \psi)$ применяются в СССР при производстве абразивных и формовочных материалов.

\section{Зависимость логарифмических гранулометрических шкал}

Из определяющих уравнений фи-, дзета-, гамма- и пси-шкал (1)-(3) и (10) можно составить систему

$$
\left\{\begin{array}{l}
x=2^{-\Phi} \\
x=10^{0,301-5}, \\
x=10^{-\frac{y}{10}} \\
x=10^{q-4}
\end{array}\right.
$$

Производя подстановки, получим

$$
\begin{cases}0,301 \varphi=\zeta-0,301, & (\varphi-\zeta) \\ 3,01 \varphi=\gamma & (\varphi-\psi) \\ 0,301 \varphi=4-\psi, & (\varphi-\psi) \\ \zeta-0,301=\frac{\gamma}{10}, & (\zeta-\psi) \\ 0,301-\zeta=\psi-4, & (\zeta-\psi) \\ \frac{\gamma}{10}=4-\psi . & (\gamma-\psi)\end{cases}
$$

(Первое из этих уравнений приводилось раньше У. Крамбейном, см. Krumbein, 1939).

Таблища I

\begin{tabular}{|c|c|c|c|c|}
\hline & Фи-шкала & Дзета-шкала & Гамма-шкала & Пси-шкала \\
\hline $\begin{array}{c}q \\
x_{0}(M \mu)\end{array}$ & $\begin{array}{l}2 \\
1\end{array}$ & $\begin{array}{c}10 \\
2\end{array}$ & $\sqrt[10]{10}=1,25$ & $\begin{array}{c}10 \\
0,0001\end{array}$ \\
\hline $\begin{array}{l}x \\
\varphi\end{array}$ & $\begin{array}{c}2^{-\varphi} \\
-\log _{2} x\end{array}$ & $\begin{array}{l}10^{0,301-5} \\
\frac{5}{0,301}-1\end{array}$ & $\begin{array}{l}10^{-\frac{10}{10}} \\
\frac{\gamma}{3,01}\end{array}$ & $\begin{array}{c}10^{\downarrow-4} \\
\frac{4-4}{0.301}\end{array}$ \\
\hline$\zeta$ & $0,301 \Phi+0,301$ & $0,301-\lg x$ & $\frac{7}{10}+0,301$ & $4,301-\psi$ \\
\hline$\gamma$ & $3,01 \varphi$ & $10(\zeta-0,301)$ & $-10 \lg x$ & $10(4-\psi)$ \\
\hline$\psi$ & $4-0,301 \varphi$ & $4,301-\zeta$ & $4-\frac{\gamma}{10}$ & $\lg x+4$ \\
\hline
\end{tabular}

Формулы, связывающие логарифмические гранулометрические шкалы

Решая каждое уравнение относительно $\varphi, \zeta, \gamma$ и $\psi$, получим зависимости (табл. 1), которые связывают все описанные выше логарифмические шкалы. Әти формулы служат для переведения размера, выраженного в единицах любой применяемой в настоящее время логарифмиче- 
ю. Maan

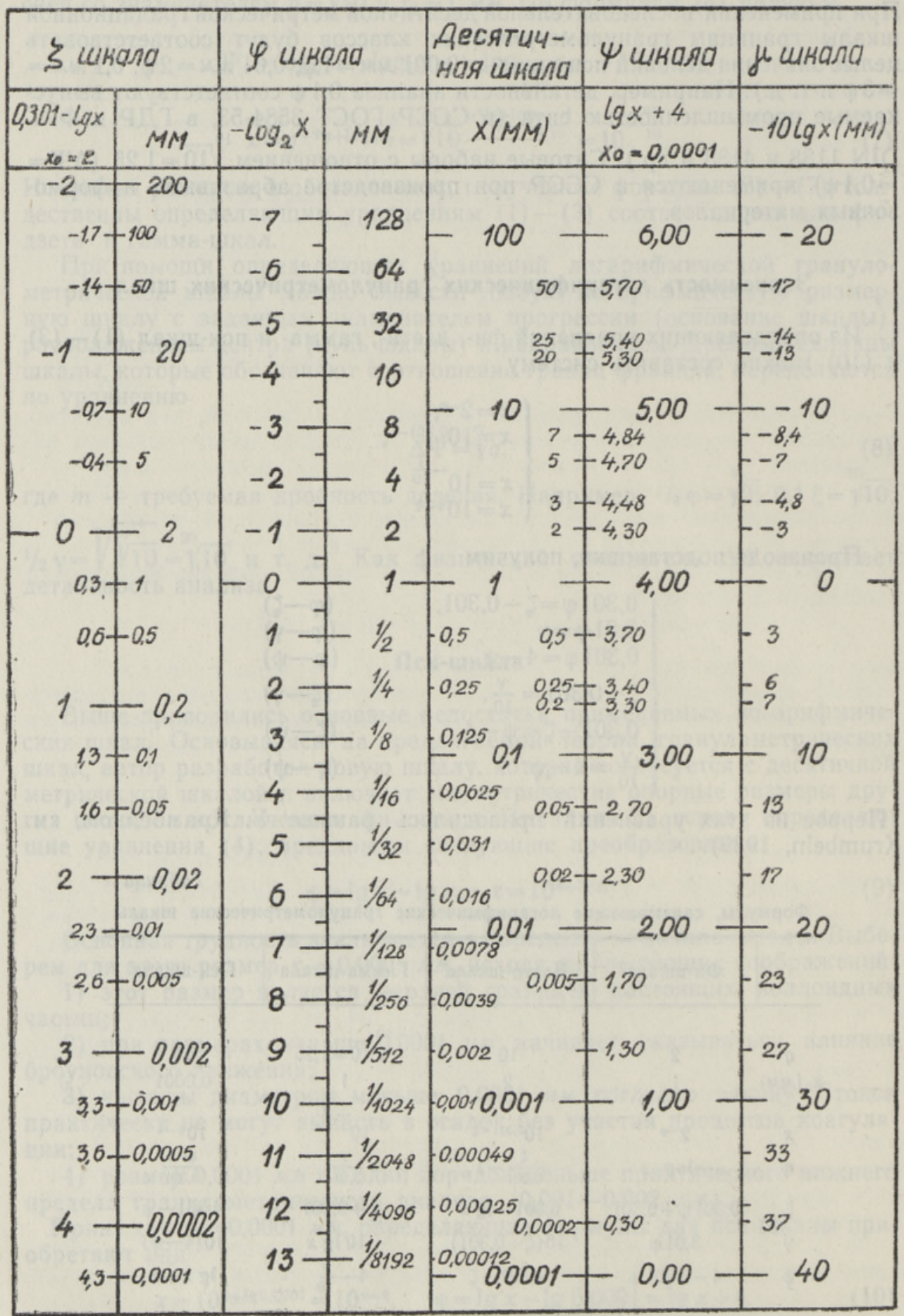

Рис. 2. Логарифмические гранулометрические шкалы (фи- и дзета-шкалы Крамбейна, гамма-шкала Батурина и пси-шкала),

Logarithmic grain size scales (Krumbein's $\varphi$ and $\zeta$ scales, Baturin's $\psi$ scale and $\psi$ scale). 
ской гранулометрической шкалы, в единицы любой другой логарифмической шкалы без промежуточного перевода в единицы линейной размерной шкалы (метрической, дюймовой и т. п.).

Таблица 2

Соотношения абсолютных значений единиц логарифмических гранулометрических шкал

\begin{tabular}{l|c|c|c|c}
\hline & $\psi$ & $\zeta$ & $\gamma$ & $\boldsymbol{\psi}$ \\
\hline $1 \psi$ & 3,322 & 0,301 & $\frac{3,01}{10}$ & 0,301 \\
$1 \zeta$ & 0,332 & 0,1 & $\frac{1}{10}$ & 0,1 \\
$1 \%$ & 3,322 & 1 & -
\end{tabular}

Рис. 3. Номограмма для перевода размеров, выраженных в миллиметрах или в единицах фи-, дзета-, гамма- или пси-шкалы, в единицы желаемой шкалы. Nomograph for translation of sizes expressed in millimetres or in units the $\varphi$, $s$, $\psi$ or $\varphi$ scales, into the units if the scale wished.

Некоторые параметры гранулометрического спектра, вычисляемые при обработке́ данных гранулометрии, выражаются в единицах длины (например, все цифровые оценки сортированности). Оценки сортированности показывают, сколько (или какую часть) единиц размерной шкалы охватывает определенная часть гранулометрического спектра. При этом абсолютные значения подобных оценок зависят от применяемой размерной шкалы. Например, приведенные ниже оценки сортированности

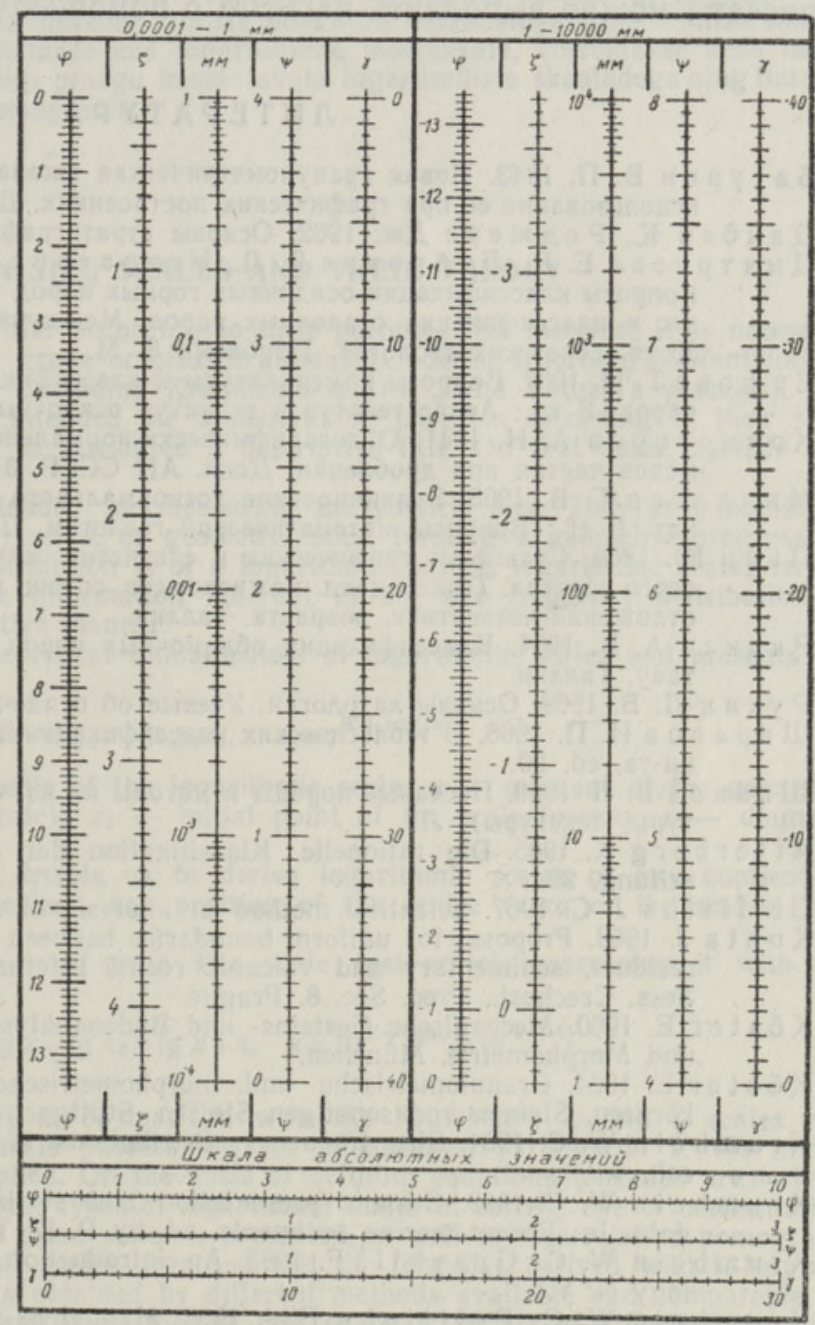

$$
\begin{aligned}
& \sigma_{1}=2,67 \varphi=0,804 \zeta=8,04 \quad \gamma=0,804 \psi, \\
& \sigma_{2}=12,0 \gamma=3,99 \varphi=1,200 \zeta=1,200 \psi, \\
& \sigma_{3}=0,181 \psi=0,602 \varphi=0,181 \zeta=1,81 \gamma
\end{aligned}
$$


равны по физическому содержанию, но различны по абсолютным значениям оценок. Цифровые коэффициенты для перевода абсолютных значений логарифмических гранулометрических шкал в другие логарифмические единицы приводятся в табл. 2. На рис. 2 дается графическое сопоставление разных логарифмических гранулометрических шкал, а на рис. 3 номограмма, служащая для перевода размеров, выраженных в миллиметрах, единицах фи-, гамма- или пси-шкалы, в единицы желаемой шкалы.

\section{Заключение}

Предлагаемая теория логарифмических гранулометрических шкал и выводимые с ее помощью следствия делают доступными и сопоставимыми все данные гранулометрии, а также графические и статистические модели, полученные с применением любых шкал. Все необходимые расчеты можно выполнить на ЭВМ с помощью программы-транслятора.

\section{ЛИТЕРА Т У Р А}

Б а т урин В. П. 1943. Новая гранулометрическая шкала для кластических осадков и использование ее при графических построениях. Докл. АН СССР, 38, № 8.

Д а н 6 а р К., Р одж е р с Дж. 1962. Основы стратиграфии. М.

Д митриева Е. В., Либрович В. Л., Некрасова О. И., Хабаков А. В. 1968. Вопросы классификации осадочных горных пород (тезисы доклада). В сб.: Генезис и классификация осадочных пород. Междунар, геол. конгр., XXIII сессия. Докл. советских геологов. Проблема 8. М.

Е ршова Г. И. 1962. Вопросы номенклатуры и классификации обломочных и глинистых пород. В кн.: Атлас текстур и структур осадочных горных пород, 1. М.

Колмогоров А. Н. 1941. О логарифмически-нормальном законе распределения размеров частиц при дроблении. Докл. АН СССР, 31, № 2.

Миддлтон Г. В. 1968 Возникновение логнормального распределения частот в осадках. В сб.: Вопросы математической геологии. Л.

П а а П Ю. 1969. Сравнение графических и статистических параметров гранулометрического спектра. Тезисы докл. межведомств. совещ. по методике изуч. терригенных отложений четвертичн. возраста. Таллин.

Р а ука с А. В. 1964. Классификация обломочных пород по гранулометрическому сос таву. Таллин.

Р ухин Л. Б. 1969. Основы литологии. Ученые об осадочных породах. Л.

Ш а р а п в И. П. 1966. О геологических классификациях. Научн. тр. Пермск. политехн. ин-та, сб. 20.

Ш в а н в В.Н 1969. Песчаные породы и методы их изучения (распространение, структуры, текстуры). Л.

Atterberg K. 1905. Die rationelle Klassifikation der Sande und Kiese. Chemikerzeitung, 29.

Griffith s J. C. 1967. Scientific method in analysis of sediments. N. Y.

$\mathrm{K}$ on t a J. 1968. Proposal for uniform boundaries between main size categories of clastic residual, sedimentary and volcanic rocks. Internat. Geol. Congr. Report 23-rd Sess. Czechosl., Proc. Sec. 8. Prague.

Köster E. 1960. Mechanische Gesteins- und Bodenanalyse. Leitfaden der Granulometrie und Morphometrie. München.

Köster E. 1964. Granulometrische und morphometrische Messmethoden an Mineralkörnern, Steinen und sonstigen Stoffen. Stuttgart.

Krumbein W. C. 1934. Size frequency distributions of sediments. J. Sediment. Petrol., 4.

Krumbein W. C. 1939. Graphic presentation and statistical analysis of sedimentary data. In: Recent marine sediments, ed. by P. D. Trask. London.

Krumbein W. C., Graybill F. 1965. An introduction to statistical models in geo$\log y$ N. Y.

Krumbein W. C., Pettijohn F. J. 1938. Manual of sedimentary petrograhpy. N. Y

$M$ üll e r G. 1964. Methoden der Sedimentuntersuchung. (Engelhardt W., Fürchtbauer H., Müller G. Sediment-Petrologie. Teil 1.) Stuttgart.

Pettijohn F. J. 1949. Sedimentary rocks. N. Y.

T a n ner W. F. 1969. The particle size scale. J. Sediment. Petrol., 39, No. 2. 
Udden J. A. 1898. Mechanical composition of wind deposits. Augustana Library publ., No. 1.

Wentworth C. K. 1922. A scale of grade and class terms for clastic sediments. J. Geol., 30.
Ннститут геологии
Академии наук Эстонской ССР
Поступила в редакцию
1/VIII 1972

O. PAAP

\section{GRANULOMEETRILISED MOOTSKAALAD JA NENDE TEOORIA}

Granulomeetrilised skaalad jaotatakse gradatsioonilisteks ehk klassifikatsioonilisteks (Uddeni, Atterbergi, Wentworthi jt. skaalad) ning mōôtskaaladeks (nende hulgas logaritmilised Krumbeini $\varphi$ ja $\zeta$ ning Baturini $\psi$-skaala).

Artiklis käsitletakse logaritmiliste mōōtskaalade teoreetilisi aluseid ja antakse valemid, mille abil saab tuletada ükskōik milliste etteantud omadustega logaritmilise skaala. Esitatud teooria alusel tuletatakse uus logaritmiline mōōtskaala, vōrreldakse seda matemaatiliselt ja graafiliselt kőigi praegu kasutatavate logaritmiliste skaaladega ning tuuakse ära neid omavahel siduv nomogramm.

\section{O. PAAP}

\section{GRAIN SIZE SCALES AND THEIR THEORY}

The grain size scales differ according to their purpose, and therefore it is necessary to differentiate between the grade or classificational (Udden's, Atterberg's, Wentworth's, etc. scales) and size scales (including Krumbein's $\varphi$ and $\zeta$ and Baturin's $\gamma$ scales). The grain-size grade scales are intended for systematizing particles according to their size, whereby each class (taxon) is attributed a descriptive title. On that basis, particle size classifications are made.

The size scales are intended for measuring the particle size. They may be linear. forming an arithmetic progression, or geometric ones, forming a geometric progression. Since the size frequency distribution is of a logarithmic nature, logarithmic scales render the physical essence of the information inherent in the size frequency distribution of sediments in the most effective manner.

The author suggests theoretical fundamentals of logarithmic scales and presents the defining equations

$$
\Psi=\log _{q} x-\log _{q} x_{0}, \quad x=q^{\Psi+\log _{q} x_{0}}
$$

where $\Psi$ - grain size in units of the logarithmic scale; $q$ - quotient of the geometric progression (base of the scale); $x_{0}$ - initial point of the scale in $\mathrm{mm} ; x$ - diameter (grain size) in $\mathrm{mm}$.

The defining equations enable us to derive logarithmic scales of any quotient of progression (base of the scales), any position of the centre (zero of the scales) and any direction.

The author proposes a new grain size scale (psi scale), expressing it with the equations

$$
\psi=\lg x-\lg x_{0}=\lg x+4, \quad x=10^{\Psi+\lg x_{0}}=10^{\Psi-4}
$$

where $x_{0}=0.0001 \mathrm{~mm}$.

The author proves that in comparison with the Krumbein's $\varphi$ and $\zeta$ scales and Baturin's $\gamma$ scale, the $\psi$ scale possess various advantages, particularly if the metric decimal classification is applied. On the basis of defining equations formulas are derived, which serve to correlate all the types of logarithmic scales in current use. A mathematical and graphical comparison of those scales is presented, as well as a correlating nomograph.

The theory of logarithmic scales proposed and the formulas derived with its help will make all the grain size data obtained by different methods available and comparable, as well as the graphical and statistical mode's created by scientists of different countries. All the necessary calculations may be effected by electronic computers, with the help of translator programmes. 